\title{
Simulation of Heat Distribution and Thermal Damage Patterns of Diode Hair-Removal Lasers: An Applicable Method for Optimizing Treatment Parameters
}

\author{
Leila Ataie-Fashtami, M.D., Afshan Shirkavand, M.Sc., ${ }^{1,2}$ Saeed Sarkar, Ph.D., ${ }^{2,3}$ \\ Mohammadreza Alinaghizadeh, M.Sc., ${ }^{4}$ Marjaneh Hejazi, Ph.D., ${ }^{2,3}$ Mohsen Fateh, M.D., M.P.H., \\ Gholamreza Esmaeeli Djavid, M.D.,' Nasrin Zand, M.D., and Hanieh Mohammadreza, M.Sc.,3
}

\begin{abstract}
Objective: We simulated the heat distribution and thermal damage patterns of diode hair-removal lasers for different spot sizes, pulse durations, and fluences as a guide for optimization. Background: Recently, the concept of thermal damage time as a reference for pulse duration has become a subject of debate. Methods: Laser-InducedTemperature-Calculation-In-Tissue (LITCIT) was used for the simulations. Skin was modeled as two homogenous layers of epidermis/dermis and two coaxial cylinders as the hair shaft/ follicle. Opto-thermal coefficients of the components and the radiant parameters of the laser (diode, $810 \mathrm{~nm}$ ) were defined. Results: At constant fluences and pulse durations, the damage occurred deeper when larger spot sizes were used. At constant pulse duration, high fluences caused significant damage to the hair follicle and epidermis. By using longer pulse durations $(\leq 400 \mathrm{~ms})$ at constant fluences, there was more effective damage to the hair follicle while sparing the adjacent epidermis and dermis. Because of the time-dependent temperature profiles, an increased pulse duration creates a moderate, gradual rise in the target's temperature. Pulse durations $>400 \mathrm{~ms}$ are accompanied by unwanted dermis damage. Conclusions: Our results show that using very long pulse durations near the tissue damage time $(\leq 400 \mathrm{~ms})$ creates better efficacy in treating unwanted hairs while avoiding unwanted damage.
\end{abstract}

\section{Introduction}

$S_{\text {nes }}^{\text {ne }}$ INCE BEING APPROVED by THE FDA in 1995, laser systems have been successfully applied as a safe and effective method for hair removal. ${ }^{1}$ Based on the theory of selective photothermolysis, hair removal is achieved by selectively depositing energy into the hair shaft and pigmented follicular epithelium in such a way that the rapid rise in temperature and subsequent heat transfer to the adjacent tissues causes only local thermal necrosis of the follicles' regenerative structures. ${ }^{2,3}$ Melanin, the principal target chromophore for hair-removal lasers, is found not only in hair shafts and follicles but also in the epidermis. ${ }^{3}$ Unfortunately, because of unavoidable absorption of laser energy by melanin in the epidermis, it is not possible to heat hair follicles without heating the epidermis, and it becomes necessary to protect the epidermis from unwanted thermal side effects. ${ }^{1}$

Hair-removal devices generally differ in their choice of wavelength, fluence, pulse duration, and spot size. These characteristics are all important and play a crucial role in both the safety and efficacy of the treatment procedure. ${ }^{2}$ To achieve effective heating of the target hair while avoiding thermal damage to the epidermis, it is critical to select the radiant parameters of laser systems properly to induce adequate thermal damage to the entire hair follicle. According to the original selective photothermolysis theory, this means choosing a proper wavelength, a proper fluence, and a pulse duration appropriate to the concept of the thermal relaxation time (TRT) (25-100 ms). ${ }^{3}$ However, since the extended theory of selective photothermolysis was proposed by Altshuler et al. in recent

\footnotetext{
${ }^{1}$ Iranian Center for Medical Lasers, Academic Center for Education, Culture and Research, Tehran, Iran.

${ }^{2}$ Research Center of Science and Technology in Medicine, Tehran University of Medical Sciences, Tehran, Iran.

${ }^{3}$ Medical Physics and Biomedical Engineering Department, Tehran University of Medical Sciences, Tehran, Iran.

${ }^{4}$ Noor Medical Imaging Center, Tehran, Iran.

A part of this study has been already presented at the American Society for Laser Medicine and Surgery Twenty-Eighth Annual Congress, Kissimmee, Florida April 2-6, 2008 and you can find it in the congress proceedings (Abstracts; No.292), and as an e- Poster http:// onlinelibrary.wiley.com/doi/10.1002/lsm.20661/abstract.
} 
years, ${ }^{3}$ choosing a pulse duration based on the concept of the thermal damage time (TDT) (200-400 ms), which is much longer than the TRT, has become the subject of much debate. ${ }^{4} \mathrm{~A}$ simulation of the thermal damage patterns from diode $(810 \mathrm{~nm})$ hair-removal lasers with long pulse durations (according to the TDT) versus short ones (according to the TRT) may shed light on how to choose the right pulse durations.

Alternatively, it is necessary to understand the basic underlying principles of tissue optics, light delivery into tissue, the resultant heat production, the heat distribution, and the thermal damage, to optimize the hair-removal process. ${ }^{5}$ Optimal laser treatment parameters have been proposed based on a mathematical analysis of light propagation in human skin using the thermal and optical properties of the follicle and its adjacent structures. To model laser-tissue interactions, many methods have been developed, including Monte Carlo based methods. Using this simulation method, we can model light propagation in a multi-layer tissue and calculate the distribution of absorbed light and generated heat within the tissue during the laser irradiation. ${ }^{5}$

The goal of our study is to investigate the effect of changes in pulse duration, fluence, and spot size of diode $(810 \mathrm{~nm})$ hair-removal lasers on the patterns of heat distribution and thermal damage in skin tissue with Laser-InducedTemperature-Calculation-In-Tissue (LITCIT) simulation software. The study aims to find the optimal parameters for diode $(810 \mathrm{~nm})$ laser photoepilation systems to achieve optimal treatment results and fewer side effects. We also hope that this simulation might offer dermatologists a better understanding of how any changes in the parameters of diode hair-removal lasers can cause thermal damage to skin.

\section{Materials and Methods}

\section{Tissue modeling}

To simulate the patterns of heat distribution and thermal damage, a model of skin must be chosen. The choice of this model is limited by the knowledge of the variables regarding the optical properties of the components in skin. ${ }^{6}$ The skin tissue is modeled by forming uniform planar layers and structures that are each defined by factors such as thickness, position, shape, index of refraction, anisotropy factor, the absorption and scattering coefficients, and thermal properties. ${ }^{7}$ We assumed that the skin surface is smooth and has a semiinfinite two-layer geometry to simulate human skin, which is composed of a top 0.1-mm-thick epidermis overlying a semiinfinite 3.9-mm-thick dermis.,8,9 Tissue volume was discretized into 3-D Cartesian grid points or voxels* where the volumetric heat production and thermal damage were calculated. ${ }^{7}$ We also modeled the hair target as two coaxial cylinders with $4 \mathrm{~mm}$ of depth: a heavily pigmented cylinder as a hair shaft and a surrounding cylinder as a hair follicle. ${ }^{3}$ The scheme of this geometry is illustrated in Fig. 1. In this figure, the protruding outer part of the hair shaft is only for easier understanding of the model. In fact, the hair shaft was considered in all simulations to end at the level of the epidermis.

We used LITCIT version 1.31 (Laser- und Medizintechnik $\mathrm{GmbH}$, Berlin, Germany). It has already been validated against some standard mathematical codes such as Monte

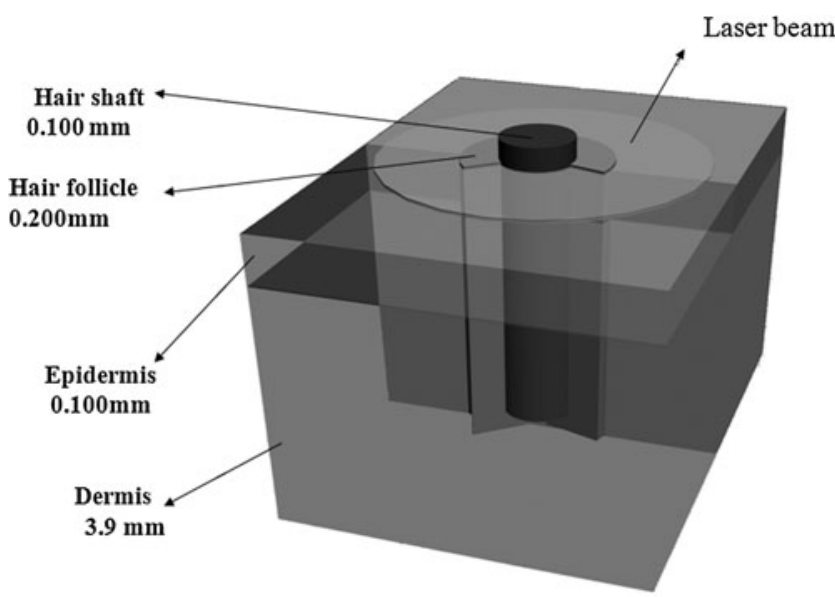

FIG. 1. The 3D-geometry of modeled tissue.

Carlo Multi-Layer (MCML) and numerical solutions of heat conduction, which in turn had previously been evaluated against experimental and clinical studies. ${ }^{10}$ This simulation program calculates and displays the heat distribution and thermal damage patterns in biological tissues during superficial and interstitial thermal treatments. The software consists of three main components, which will be discussed below. The structure of LITCIT combines these components through an algorithm to calculate the energy and heat distribution of the laser radiation in addition to the thermal damage. ${ }^{11}$

Calculation of the light distribution. LITCIT is a Monte Carlo based method to calculate the light intensity inside the irradiated tissue. The propagation of laser in biological tissue and its transformation into thermal energy caused by absorption of the photons is governed by optical properties such as the absorption coefficient $\mu_{\mathrm{a}}\left(\mathrm{mm}^{-1}\right)$, the scattering coefficient $\mu_{s}\left(\mathrm{~mm}^{-1}\right)$, the anisotropy factor $\mathrm{g}$ and the refractive index $\mathrm{n} .{ }^{11,}$ 12 We calculated the absorption coefficient of the epidermis according to Jacques' Equation. In this work, we assumed that the fraction of melanosome in the epidermis $f_{m e l}=11 \%$ for moderately pigmented skin, which corresponds to skin type 3. The total absorption coefficient of the epidermis $\left(\mu_{\text {aepi }}\right)$ combines the baseline skin absorption and the melanin absorption and is calculated according to the following equation:

$$
\mu_{\text {a.epi }}=\left(f_{\text {mel }} \times \mu_{\text {a.mel }}\right)+\left(1-f_{\text {mel }}\right)\left(\mu_{\text {a.baseline }}\right) \mathrm{cm}^{-1}
$$

where

$$
\begin{aligned}
& \mu_{\text {a.mel }}=6.6 \times 10^{11}\left(\lambda^{-3.33}\right) \mathrm{cm}^{-1} \\
& \mu_{\text {a.baseline }}=0.244+85.3 \exp \left(-\frac{\lambda-154}{66.2}\right) \mathrm{cm}^{-1}
\end{aligned}
$$

and $\lambda$ is the wavelength of laser in nanometers. ${ }^{12}$ We also assumed that the skin components have a uniform refractive index and that therefore no refraction or reflection occurs when light passes from one component to another. Additionally, we assumed that the scattering coefficients and anisotropy factors of the skin constituents were all the same

*Volumetric Pixel. 
Table 1. Optical Properties of Various Skin Components Used in Our Study $(\lambda=810 \mathrm{NM})$

\begin{tabular}{lcccc}
\hline Components & $\mu_{a}\left(\mathrm{~mm}^{-1}\right)$ & $\mu_{s}\left(\mathrm{~mm}^{-1}\right)$ & $g$ & $\mathrm{n}$ \\
\hline Epidermis & 1.5 & 5 & 0.87 & 1.37 \\
Dermis & 0.023 & 5 & 0.87 & 1.37 \\
Hair follicle & 3.5 & 5 & 0.87 & 1.37 \\
Hair shaft & 1.5 & 5 & 0.87 & 1.37
\end{tabular}

$\mu_{a}\left(m^{-1}\right)$ : Absorption coefficient, $\mu_{s}\left(m^{-1}\right)$ : scattering coefficient, $\mathrm{g}$ : anisotropy factor, $\mathrm{n}$ : refractive index.

as in previous works. ${ }^{5,13}$ The optical properties for each skin component are listed in Table 1.

Calculation of the heat conduction. In LITCIT, the heat deposition is individually calculated for each voxel after each time interval. The temperature rise $\Delta T(x, y, z)$ is determined by the amount of converted energy $\Delta E(x, y, z)$ :

$$
\mathrm{dQ}=\operatorname{mcdT} \rightarrow \Delta T(x, y, z)=\frac{\Delta E(x, y, z)}{\rho \cdot C_{p} \cdot V_{\text {voxel }}}
$$

where $\rho$ and $C_{p}$ are thermal properties of the tissue. For calculations of heat transfer, a numerical solution of the corresponding differential equation is solved by the method of finite differences. Another thermal parameter for these calculations known as the heat conduction coefficient, $k$, is needed. All these calculations are run automatically by the software. From physical references, the thermal properties of tissue can be calculated approximately depending upon the water content of the tissue $(w))^{5,13}$

$$
\begin{array}{rlrl}
\kappa & =4.19(0.133+1.36 w) \times 10^{-3} & & (\mathrm{~W} / \mathrm{cmK}) \\
C_{p} & =4.19(0.37+0.63 w) & & (\mathrm{J} / g K) \\
\rho & =1.3-0.3 w & \left(\mathrm{~g} / \mathrm{cm}^{3}\right)
\end{array}
$$

We assumed that $w=0.50$ for the epidermis and hair and $w=0.75$ for the dermis. ${ }^{5}$ Thermal properties used in the modeling of heat transfer including those for the skin components calculated using Equations 5-7 are summarized in Table 2. We assumed that the initial temperatures of the skin model at the surface and the bottom were $30^{\circ} \mathrm{C}$ and $37^{\circ} \mathrm{C}$ respectively. We also assumed that the initial temperature of the contact-cooling sapphire was $5^{\circ} \mathrm{C}$ as is given for diode hair-removal systems. We neglected heat transfer caused by blood perfusion because those effects are not important on the short time scales (hundreds of milliseconds) used in this study. ${ }^{2}$

Calculation of tissue damage. Any area in which the temperature increased $>60 C^{\circ}$ underwent coagulation, and

Table 2. Thermal Properties of Various Skin Components Used in Our Study

\begin{tabular}{lclc}
\hline Components & $\kappa\left({ }^{W} / \mathrm{cmK}\right)$ & $\rho\left(g / \mathrm{cm}^{3}\right)$ & $\mathrm{C}_{p}\left({ }^{\mathrm{J}} /{ }_{\mathrm{gK}}\right)$ \\
\hline Epidermis & 0.0034 & 1.1497 & 2.789 \\
Dermis & 0.0048 & 1.075 & 3.488 \\
Hair follicle & 0.0034 & 1.1497 & 2.789 \\
Hair shaft & 0.0034 & 1.1497 & 2.789 \\
\hline
\end{tabular}

the affected cells are presumed to have died immediately or after a certain period of time. ${ }^{13}$ As an approximation, the temperature behavior of cells can be described by a damage integral $\Omega(T, t)$ based on the Arrhenius formulae. This integral calculates the state of protein denaturation as a rate equation: ${ }^{5,11,13}$

$$
\Omega(T, t)=A \int_{t_{i}}^{t_{f}} \exp \left(\frac{-E}{R T}\right) d t
$$

This integral describes the probability of tissue destruction. Skin tissue is considered to be irreversibly damaged when $\Omega \geq 1$ or the thermal damage is $>63 \%{ }^{8}$ In this integral, $R$ is the universal gas constant $(8.314 \mathrm{~J} / \mathrm{mol} . \mathrm{K}), t$ is the time of irradiation, and $A$ and $E$ are the Arrhenius constants with values of $\left(3.1 \times 10^{98} \mathrm{~s}^{-1}\right)$ and $\left(6.3 \times 10^{5} \mathrm{~J} / \mathrm{mol}\right)$, respectively. 5,13

\section{Laser systems}

In this study, we compared two photoepilation diode $(810 \mathrm{~nm})$ laser systems. One system had a $10-\mathrm{mm}$ spot size, a fluence of $0.1-100 \mathrm{~J} / \mathrm{cm}^{2}$, and pulse durations of $50-1200 \mathrm{~ms}$; and the other one had a $5-\mathrm{mm}$ spot size, a fluence of $0.1-$ $200 \mathrm{~J} / \mathrm{cm}^{2}$, and pulse durations of $5-400 \mathrm{~ms}$. We modeled the laser beam in terms of pulse durations $(50,100,150,180,200$, 300 and $400 \mathrm{~ms})$, various fluences $(20,30,40,50,55$ and $60 \mathrm{~J} /$ $\mathrm{cm}^{2}$ ) and two spot sizes (5 and $10 \mathrm{~mm}$ ). In addition, we assumed that the laser-beam energy was incident perpendicularly to the skin's surface.

\section{Results}

In this section, the results of the temperature profiles, heat distributions, and thermal damage simulation patterns in skinhair tissues at the end of laser pulse irradiation are presented. In addition, the effect of various fluences, spot sizes, and pulse durations are checked on all of the simulated patterns. All temperatures that are discussed are in degrees Celsius.

\section{Effect of beam diameter}

By using a larger spot size in contrast to smaller spot sizes, there is a more significant temperature rise at all depths of the hair follicle, but especially in the deeper parts at depths of $4 \mathrm{~mm}$ (Fig. 2a and b). In other words, when a larger spot size is used, a greater amount of heat is distributed to both the deep part of the hair follicle and the adjacent epidermis, as is indicated by the deep blue color in the graphs. As a result, there is more thermal damage in both of the deep part of the hair follicle and the epidermis when larger spot sizes are used (Fig. 3a).

\section{Effect of fluence}

Our results show that by increasing the laser fluence, temperatures rise drastically both in the epidermis and dermis, although the phenomenon is more prominent in the epidermis.

As is seen in Fig. 2 (c and d), for a constant spot size $(10 \mathrm{~mm})$ and pulse duration $(200 \mathrm{~ms})$, higher fluences cause a greater temperature rise at given depths $(0.1$ and $2 \mathrm{~mm})$ in the tissue volume. With a fluence of $60 \mathrm{~J} / \mathrm{cm}^{2}$ in contrast to 

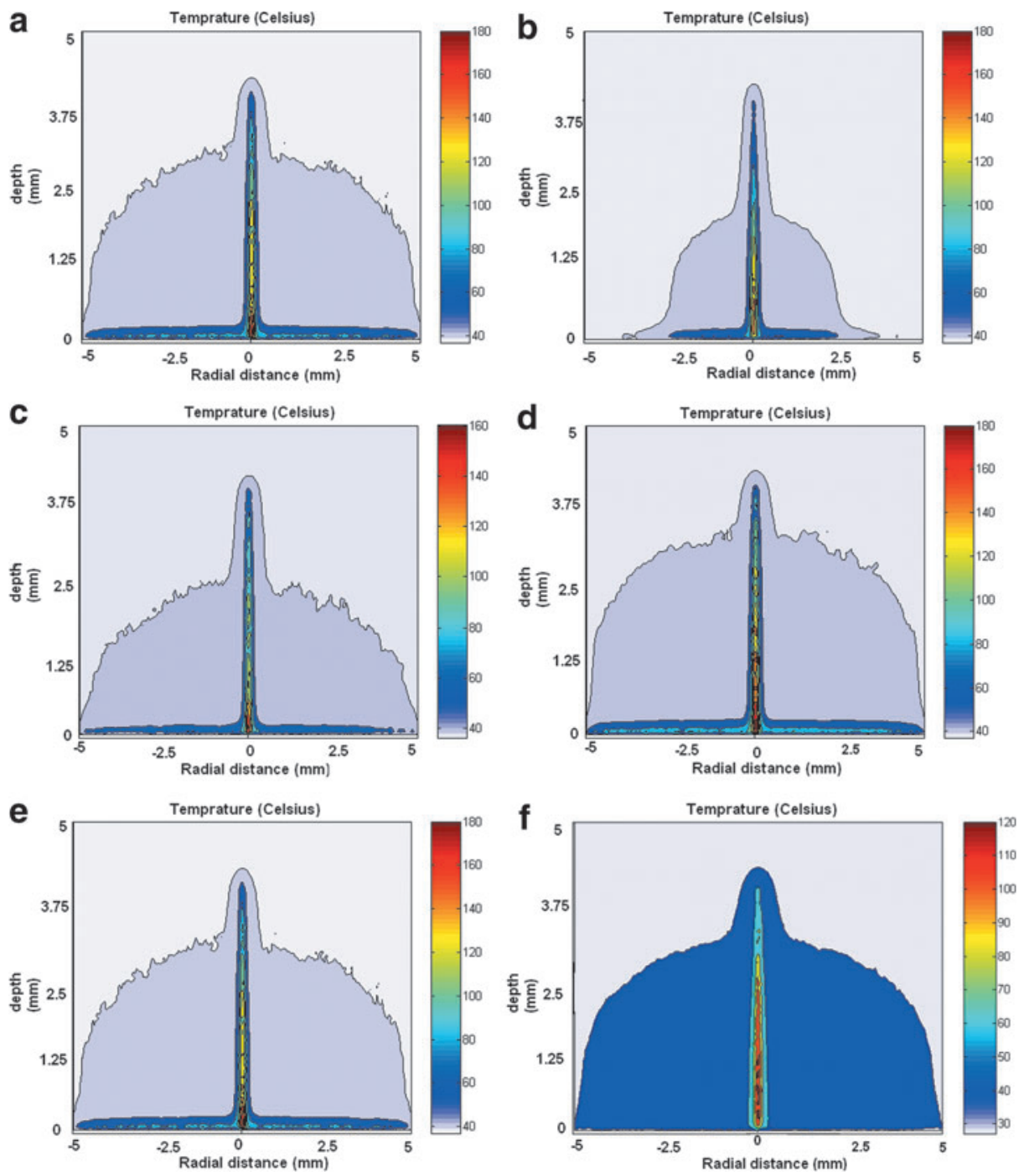

FIG. 2. Heat distribution at the end of 810 -nm laser pulse irradiation with (a) pulse duration $200 \mathrm{~ms}$, fluence $50 \mathrm{~J} / \mathrm{cm}^{2}$, spot sizes $10 \mathrm{~mm}$; (b) pulse duration $200 \mathrm{~ms}$, fluence $50 \mathrm{~J} / \mathrm{cm}^{2}$, spot sizes 5mm; (c) pulse duration $200 \mathrm{~ms}$, fluence $40 \mathrm{~J} / \mathrm{cm}^{2}$, spot sizes $10 \mathrm{~mm}$; (d) pulse duration $200 \mathrm{~ms}$, fluence $55 \mathrm{~J} / \mathrm{cm}^{2}$, spot sizes $10 \mathrm{~mm}$; (e) pulse duration $200 \mathrm{~ms}$, fluence $50 \mathrm{~J} / \mathrm{cm}^{2}$, spot sizes $10 \mathrm{~mm}$; and (f) pulse duration $400 \mathrm{~ms}$, fluence $50 \mathrm{~J} /$ $\mathrm{cm}^{2}$, spot sizes $10 \mathrm{~mm}$.
$40 \mathrm{~J} / \mathrm{cm}^{2}$, there is a more significant amount of heat $\left(65^{\circ} \mathrm{C}\right.$ versus $50^{\circ} \mathrm{C}$, respectively) distributed to both the hair follicle (hair shaft and follicle) and the epidermis.

Figure $3 \mathrm{~b}$ shows that there is an increased amount of thermal damage both to the hair follicle and to the adjacent epidermis, for increased fluence. This considerable thermal damage is caused by the greater amount of total energy that is delivered to the tissue volume while using higher fluences.

\section{Effect of pulse duration}

The simulations show that longer pulse durations cause an effective temperature rise in the deep part of the hair follicle, whereas the epidermis experiences an insignificant rise in temperature. As is shown in Fig. 2 (e and $\mathrm{f}$ ), a pulse duration of $200 \mathrm{~ms}$ is accompanied by a heat level of $\sim 60^{\circ} \mathrm{C}$ in the adjacent epidermis versus the $40^{\circ} \mathrm{C}$ level created by a pulse duration of $400 \mathrm{~ms}$. This occurs despite the fact that there is no significant difference between these two pulse durations in terms of the effective heat distribution in the hair follicles.

Therefore, using longer pulse durations up to $400 \mathrm{~ms}$ is accompanied by effective thermal damage to the hair follicle, while preserving the epidermis as expected (Fig. 3c), although excessive widening of the pulse duration seems to be accompanied by unwanted thermal damage to the adjacent dermis (Fig. 4).

Figure 5 ( $\mathrm{a}$ and $\mathrm{b}$ ) shows that whereas laser irradiation with short pulse durations produces a sharp, high-peaked and short-lived temperature rise in the target tissue, longer pulse durations are accompanied by a gradual and moderate rise in temperature that is enough to achieve an optimum temperature rise (up to $100^{\circ} \mathrm{C}$ ).

\section{Discussion and Conclusions}

In this study, we simulated the effects of laser treatment parameters (spot size, fluence, and pulse duration) on the temperature profile, heat distribution, and thermal damage patterns of hair and skin tissues. Our results showed that larger spot sizes and higher fluences produce a greater amount of distributed heat and thermal damage in both the hair follicle and the epidermis. The thermal damage to the hair follicle was found to occur deeper when larger spot sizes were used. We observed that at a constant fluence and spot size with longer pulse durations (up to $400 \mathrm{~ms}$ ), the heat distribution and thermal damage are more effective, damaging the hair follicle while sparing the adjacent epidermis and dermis. Because of the time-dependent temperature 
a

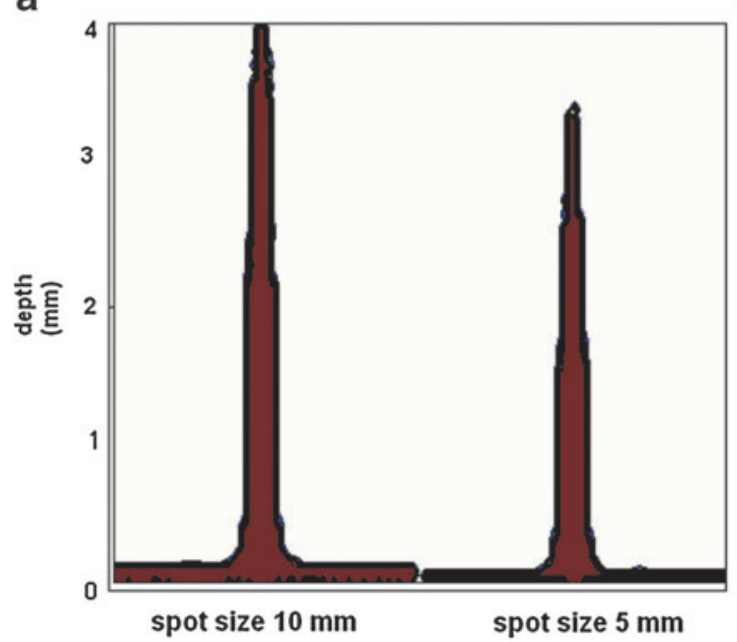

b

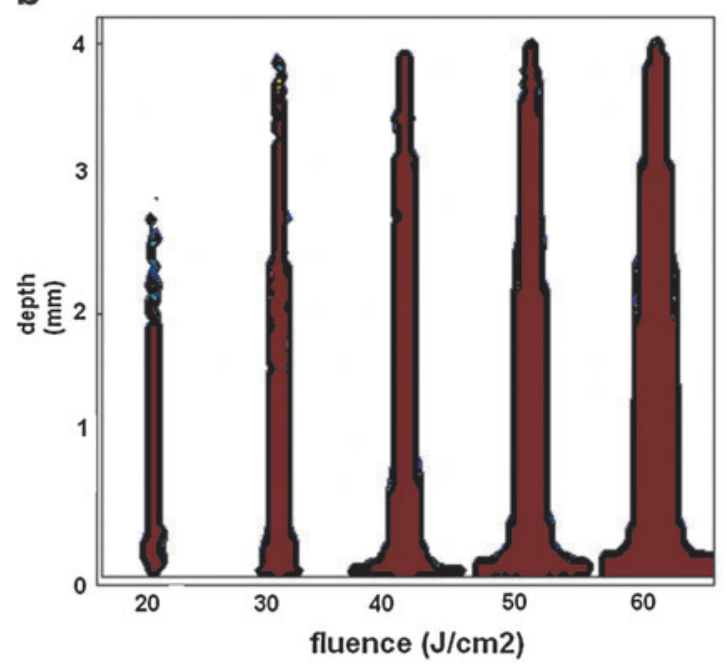

C

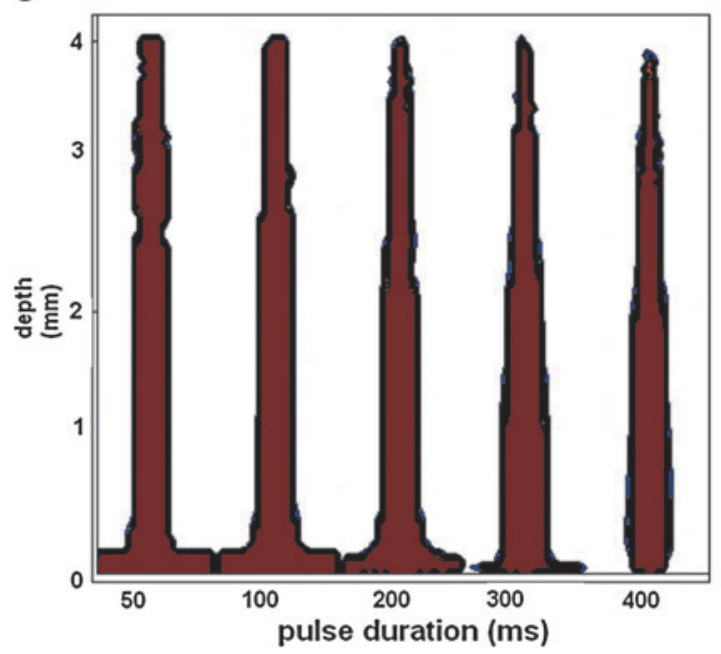

damage (\%)

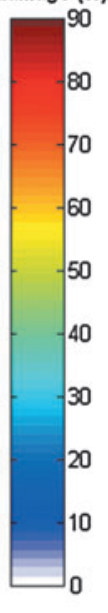

FIG. 3. Thermal damage pattern of single hair follicle and adjacent epidermis at the end of 810 -nm laser pulse irradiation with (a) pulse duration $(200 \mathrm{~ms})$ and fluences $50 \mathrm{~J} / \mathrm{cm}^{2}$ for two spot sizes, $10 \mathrm{~mm}$ and $5 \mathrm{~mm}$; (b) $10-\mathrm{mm}$ spot size and 200-ms pulse duration, for different fluences; and (c) $10-\mathrm{mm}$ spot size and fluence $50 \mathrm{~J} / \mathrm{cm}^{2}$ for different pulse durations.

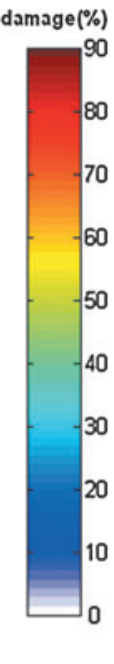

damage(\%)

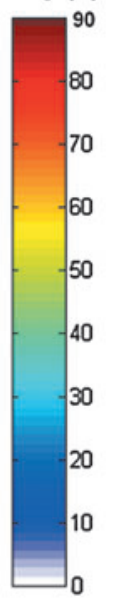

profiles, a large pulse width causes a moderate and gradual rise in target tissue temperature. However, pulse durations $>400 \mathrm{~ms}$ are accompanied by unwanted damage to the adjacent dermis.

Hair removal using laser systems is based on selective photothermolysis principles. According to the original selective photothermolysis theory (Anderson and Parrish ${ }^{15}$ ), the optimum pulse duration should be equal to or less than the thermal relaxation time of the target tissue (e.g., $100 \mathrm{~ms}$ for a hair follicle). According to the extended theory of selective photothermolysis (Altshuler et $\mathrm{al}^{3}{ }^{3}$ ), however, the optimum pulse duration for hair removal lasers or vascular lasers should be near the thermal damage time of the target tissue. The thermal damage time is much longer than the thermal relaxation time but has a rough relationship to it. ${ }^{3}$ This new concept has caused much debate in recent years. ${ }^{4}$ Although clinical data show that there would be higher efficacy and safety in hair removal using newer laser systems with longer pulse durations, ${ }^{14}$ mathematical and simulation data that are in agreement with these clinical findings seem to be missing. Our study aims to fill this crucial gap.

In this study, we deemed it necessary to investigate how to optimize the treatment results of diode hair-removal lasers by adjusting the laser parameters including spot size, fluence, and pulse duration in a simulation model. We hope that this simulation can offer dermatologists a better understanding of how changes in the parameters of diode hair removal lasers can affect the skin and hair in terms of thermal damage.

In this study, LITCIT software was used for the simulations. Because this is the first time that LITCIT was used in the field of laser hair removal, validating the software results against standard mathematical methods was crucial. Previous validation results have demonstrated its accuracy and the possibility for hair removal simulation. ${ }^{10}$

Similarly, there have been some comments on the increased thermal damage to the epidermis caused by using high fluences, because of the higher level of heat production. ${ }^{5,8}$ Our 

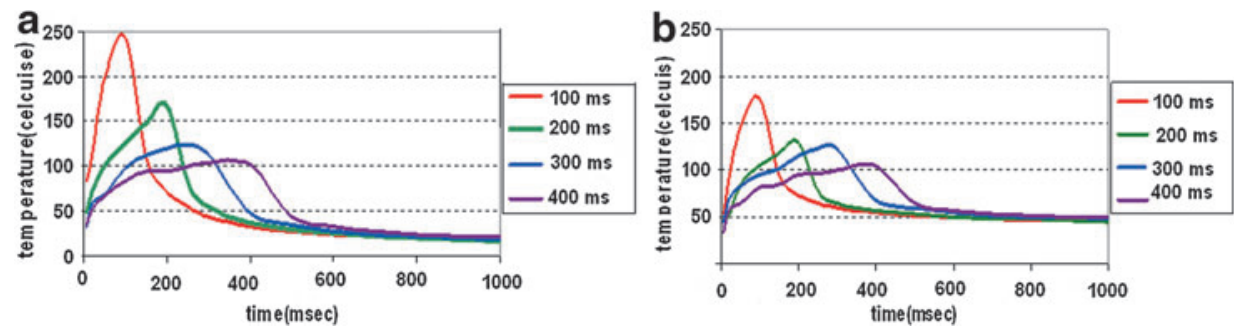

FIG. 5. Time dependent temperature profiles for 810 $\mathrm{nm}$ diode laser beam with constant spot size $(10 \mathrm{~mm})$ and constant fluence $(50 \mathrm{~J} /$ $\mathrm{cm}^{2}$ ) with different pulse durations for two depths: (a) $0.1 \mathrm{~mm}$, and (b) $2 \mathrm{~mm}$. simulation results agree with previous clinical data. As is seen in Fig. 2 (c and d), there was more significant thermal damage to both the epidermis and the hair follicle when using a higher fluence, because of the higher heat distribution in tissue. Therefore, it does not seem that increasing the fluence to achieve a better clinical outcome is an appropriate solution in light of its inevitable concomitant epidermal damage. As a result, other laser parameters should be considered, to improve efficacy.

Some studies have shown that longer pulse durations are more effective at preserving the epidermis from unwanted thermal damage. ${ }^{1,12,16,17-20}$ Our results show that short pulse durations cause irreversible thermal damage to the epidermis. ${ }^{3}$ This damage might be because of the relatively little amount of heat lost through the epidermis into the contactcooling sapphire. Instead, with longer pulse durations (up to $400 \mathrm{~ms}$ ) the epidermal protection is enhanced because of greater heat loss through the epidermis into the cooling sapphire, whereas the same effective thermal damage to the hair follicle occurs (Fig. 3c).

On the other hand, it seems that excessive increases in the pulse duration are accompanied by unwanted thermal damage to the adjacent dermis (Fig. 4). To address this, we modeled the treatment parameters that Rogachefsky et al. used in their clinical study to evaluate the side effects and efficacy of treatment of unwanted hairs using a new super-long pulse diode laser (200-1000 ms) in 2002. ${ }^{14}$ According to Fig. 10, the most reliable thermal damage pattern is achieved by the combination of a pulse duration of $400 \mathrm{~ms}$ and a fluence of $46 \mathrm{~J} / \mathrm{cm}^{2}$ (power $=90 \mathrm{~W}$ ) because the follicle volume is damaged thoroughly in this pattern whereas the adjacent epidermis and dermis tissues are spared. Using a pulse duration of $200 \mathrm{~ms}$ and a fluence of $23 \mathrm{~J} / \mathrm{cm}^{2}$ (power $=90 \mathrm{~W}$ ), the follicle cannot be damaged completely. We also observed that with a $500 \mathrm{~ms}$ pulse duration and a fluence of $57 \mathrm{~J} / \mathrm{cm}^{2}$, or a pulse duration of $1000 \mathrm{~ms}$ and a fluence of $115 \mathrm{~J} / \mathrm{cm}^{2}$ (power $=90 \mathrm{~W}$ ), there was a considerable increase in thermal damage to the adjacent dermis. ${ }^{14}$

Similar clinical studies have confirmed such an outcome. For example, a clinical trial was conducted to evaluate the safety and effectiveness of a new 400-ms extended-pulse lightsheer diode hair-removal laser in 2003. It was concluded that the pulse width of $400 \mathrm{~ms}$ enabled the safety and efficacy of the lightsheer in even the darkest skin types for longerterm hair reduction. Moreover, higher fluences with extended pulse durations in an $800-\mathrm{nm}$ diode laser can be safely applied even on darker skins to achieve complete and permanent hair reduction without the risk of adverse thermal damage. ${ }^{21}$ Likewise, we refer to a clinical study that was conducted in 2004 to assess the efficacy of a long pulse MYTHOS diode laser (40-400 ms) for permanent hair removal. It was concluded that long pulse ( $40-400 \mathrm{~ms})$ modes enabled the clinician to extend the clinical capabilities to treat different skin and hair types. Clinical experience demonstrated a high degree of safety and efficacy in patients with skin types $1-4 .^{22}$

Our study suggests that when using a diode laser for hair removal, long pulse durations up to $400 \mathrm{~ms}$ are more effective for an optimum treatment of unwanted hairs. However, with pulse durations $>500 \mathrm{~ms}$ the thermal damage to the adjacent dermis increases rapidly (Fig. 5). According to our simulation results, it seems that when larger spot sizes are available for the treatment process, lower fluences need to be delivered to the target for a given amount of power. ${ }^{23}$ Because of an inverse relationship between fluence and area, a $10-\mathrm{mm}$ spot size with fluences in the range of $40-50 \mathrm{~J} / \mathrm{cm}^{2}$ is more reliable and effective. With a smaller spot size, higher fluences may be needed to produce the same thermal effect, which is something we would not recommend, because of the high risk of thermal damage to the epidermis.

Our study does suffer from some limitations; for example, we neglected blood perfusion in the skin because of the short time scales (hundreds of milliseconds). We assumed that the skin surface was smooth, and all back reflections from the sapphire were neglected. We assumed a constant absorption coefficient for hair, but in fact it differs according to the hair color. We also ran the simulations for a constant hair diameter and depth and only for one skin type (type 3). We assumed that the laser beam profile is flat and also that the efficiency of heat production from the laser absorbed energy is $100 \%$. Our tissue model in this study consisted of one single hair structure. In reality, hair density may be different from patient to patient or from site to site in an individual patient. Despite these shortcomings, our results can give clinicians an approximate image of what is happening during laser hair removal. It is certainly necessary to simulate the heat distribution and thermal damage of a group of adjacent hairs and for various skin types and various hair colors. It is therefore wise to suggest that the parameters mentioned previously should be used with caution. To investigate the importance of using longer pulses at a variety of wavelengths and also for different skin types and hair conditions such as color, depth, and diameter, more extensive simulations and clinical studies are suggested. A simulation study for modeling thermal damage patterns related to the skin models containing more than one follicle and for various skin types or hair colors is in progress in our research group. 


\section{Acknowledgments}

This study was conducted under a grant from the Research Center for Science and Technology in Medicine, Tehran University of Medical Sciences. The authors thank Dr. Shahram Akhlaghpoor for his invaluable cooperation in providing the LITCIT software during the performance of this study.

\section{Author Disclosure Statement}

No conflicting financial interests exist.

\section{References}

1. Manestien, D., Pourshagh, M., and Anderson R.R. (2001). Effect of fluence and pulse duration for flash lamp exposure on hair follicles. Proceedings of the $21^{\text {st }}$ Annual Meeting of the American Society for Laser Medicine and Surgery, Burlington, MA.

2. Tunnell, J.W., Wang, L.V., and Anvari, B. (2003). Optimum pulse duration and radiant exposure for vascular laser therapy of dark port-wine skin: a theoretical study. Appl. Opt. 42, 1367-1378.

3. Altshuler, G.B., Anderson, R.R., Manstein, D., Zenzie, H.H., and Smirnov M.Z. (2001). Extended theory of selective photothermolysis. Lasers Surg. Med. 29, 416-432.

4. Ross, E.V. (2001). Extended theory of selective photothermolysis: a new recipe for hair cooking? Lasers Surg. Med. 29, 413-415.

5. Klavahn, K.G., and Green, D. (2002). Importance of cutaneous cooling during photo-thermal epilation: theoretical and practical consideration. Lasers Surg. Med. 31, 97-105.

6. Smithies, D.J., and Butler, H.P. (1995). Modeling the distribution of laser light in port-wine stains with the Monte Carlo method. Phys. Med. Biol. 40, 701-731.

7. Ross, E.V., and Domankevitz, Y. (2005). Laser treatment of leg veins: physical mechanisms and theoretical considerations. Lasers Surg. Med. 36, 105-116.

8. Dai, T., Pikkula, B.M., Wang, L.V., and Anvari, B. (2004). Comparison of human skin optothermal response to near infrared and visible laser irradiation: a theoretical investigation. Phys. Med. Biol. 49, 4861-4877.

9. Steiner, R., Russ, D., Falkenstein, W., and Keinle, A. (2001). Optimization of laser epilation by simulation of the thermal laser effect. Laser Phys. 11, 146-153.

10. Shirkavand, A., Sarkar, S., Hejazi, M., Ataie-Fashtami, L., and Alinaghizadeh, M.R. (2007). Evaluation of LITCIT software for thermal simulation of superficial lasers like hair removal Lasers Indian J. Dermatol. 52, 145-149.

11. LITCIT for windows: Therapy planning system for thermal laser application. Laser- und Medizin-Technologie $\mathrm{GmbH}$ B. Technical Reference Manual; Version 1.31. Berlin; 2000.
12. Gemert, V. M. J. C., Jacques, S. L., Sterenborg, H. J. C. M., and Star, W. M. (1989). Skin Optics. IEEE. Trans. Biomed. Eng. 36, 1146-1154.

13. Niemz, M. H. (1996). Laser tissue interactions, fundamentals and applications. Berlin, Heidelberg Springer-Verlag, pp. 10-120.

14. Rogachefsky, A.S., Silapunt, S., and Goldberg, D.J. (2002). Evaluation of a new super-long-pulsed $810 \mathrm{~nm}$ diode laser for the removal of unwanted hair: the concept of thermal damage time. Dermatol. Surg. 28, 410-414.

15. Anderson, R.R., and Parrish, J.A. (1983). Selective photothermolysis: precise microsurgery by selective absorption of pulsed radiation. Science 220, 524-527.

16. Sadick, N.S., and Prieto, V.G. (2003). The use of a new diode laser for hair removal. Dermatol. Surg. 29, 30-34.

17. Altshuler, G.B., Zenzie, H.H., Erofeev, A.V., Smirnov, M.Z., Anderson, R.R., and Dierickx, C. (1999). Contact cooling of the skin. Phys. Med. Biol. 44, 1003-1023.

18. Adrian, R.M., and Shay, K.P. (2000). $800 \mathrm{~nm}$ diode laser hair removal in African American patients: a clinical and histological study. J. Cutan. Laser. Ther. 2, 183-190.

19. Campos, V.B., Dierickx, C.C., Farinelli, W.A., Lin, T.Y., Manuskiatti, W., and Anderson, R.R. (2000). Hair removal with 800-nm pulsed diode laser (abs). J. Am. Acad. Dermatol. 43, 442-447.

20. Fiskerstrand, E.J., Svaasand, L.O., and Nelson, J.S. (2003). Hair removal with long pulsed diode lasers: a comparison between two systems with different pulse structures. Lasers Surg. Med. 32, 399-404.

21. Trelles, M A., Urdiales, F., and Al-Zarouni, M. (2009). Hair structures are effectively altered during $810 \mathrm{~nm}$ diode laser hair epilation at low fluencies. J. Dermatolog. Treat. 20(4), 1-4.

22. Volvovsky, M. (2004). High-power (500 W) short (20-200 ms) and long (40-400 ms) pulse diode laser for permanent hair removal, Class Clinic, Sheba Medical Center, Tel Hashomer, Israel. Available at: http://www.msq.co.il/pdf/white_ paper_mythos.pdf

23. Baumler, W., Scherer, K., Abeles, C., Neff, S., Lantharel, M., and Szeimies, R.M. (2002). The effect of different spot sizes on the efficacy of hair removal using a long-pulsed diode laser, Dermatol. Surg. 28, 118-121.

24. Incropera, F.P., and Dewitt D. P. (1990). Fundamentals of heat and mass transfer. New York: John Wiley \& Sons.

Address correspondence to: Saeed Sarkar, Ph.D.

Research Center of Science and Technology in Medicine (RCSTIM) Tehran University of Medical Sciences Tehran, Iran 1419733141

E-mail: sarkar@sina.tums.ac.ir 
